\title{
Low-Dose Eltrombopag in a Patient with Chronic Idiopathic Thrombocytopenic Purpura Post Sleeve Gastrectomy
}

\author{
Mohanad A. Ahmed ${ }^{a}$ Mohamad A. Yassin ${ }^{b}$ \\ aDepartment of Medical Education, Hamad Medical Corporation, Doha, Qatar; \\ bepartment of Medical Hematology and Oncology, Hamad Medical Corporation, \\ Doha, Qatar
}

\section{Keywords}

Eltrombopag $\cdot$ Idiopathic thrombocytopenic purpura $\cdot$ Sleeve gastrectomy

\begin{abstract}
Idiopathic thrombocytopenic purpura (ITP) is a disease in which the immune system attacks platelets and causes decrease in its number exposing the patient to risk of bleeding. It is diagnosed by exclusion. Eltrombopag is a thrombopoietin receptor agonist which is used as second-line treatment for patients with ITP. The usual starting dose is $25 \mathrm{mg}$ daily and maintenance dose is $75 \mathrm{mg}$ daily. Little is known about the dose of eltrombopag in patients with sleeve gastrectomy since reduction in the amount of functioning gastrointestinal tract after gastric bypass surgery leads to decreased time to drug absorption and reduced drug bioavailability. Here we are reporting a 46-year-old female with ITP and sleeve gastrectomy who responded to low-dose eltrombopag $25 \mathrm{mg}$ every other day, which is equivalent to $12.5 \mathrm{mg}$ daily, and maintained adequate platelet counts on this dose.

\section{Introduction}

The pathogenesis of idiopathic thrombocytopenic purpura (ITP) is incompletely understood. Reduced platelet lifespan due to antibody-mediated destruction is the predominant hypothesis; however, other mechanisms are likely important, including autoreactive cytotoxic T cells, as well as humoral and cellular autoimmunity directed at megakaryocytes, causing impaired platelet production. The principal mechanism is thought to involve specific immunoglobulin G (IgG) autoantibodies produced by the patient's B cells, most often directed 
Fig. 1. Changes in platelet count over time from September 2019 to February 2020.

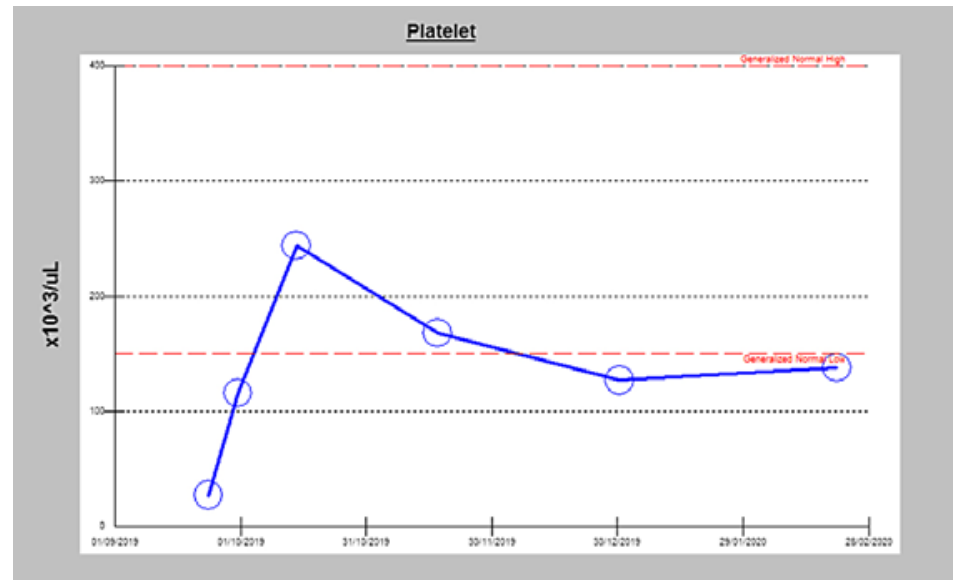

against platelet membrane glycoproteins such as GPIIb/IIIa [1, 2]. Many patients with ITP are asymptomatic. For those who do have symptoms, these are primarily related to thrombocytopenia and bleeding, but patients can also experience fatigue and a reduced quality of life. ITP is a diagnosis of exclusion. It is defined as isolated thrombocytopenia (platelet count $<100,000 / \mathrm{mL}$ ) without anemia or leukopenia and without another apparent cause of the thrombocytopenia $[1,3]$. The goal of ITP therapy is to provide a safe platelet count to prevent clinically important bleeding, rather than to normalize the platelet count $[1,3]$. There are three principal choices of second-line treatment, which differ in their mechanism of action, efficacy, and risks: splenectomy, rituximab, and thrombopoietin receptor agonists $[3,4]$.

Eltrombopag is a thrombopoietin receptor agonist. The recommended daily dose is 50 mg daily to maintain a platelet count that reduces the risk of spontaneous bleeding [5].

The effect of obesity-related surgery on the eltrombopag function was not studied to the extent of our knowledge.

\section{Case Report/Case Presentation}

We present a case of a 46-year-old female, a known case of diabetes type 2 and hypothyroidism. The patient had sleeve gastrectomy in 2014. She presented with thrombocytopenia on 2015, and her platelet count was 37 (normal 100-400). The patient was asymptomatic. At that time, workup was done and revealed normal CBC. Her peripheral smear showed thrombocytopenia. She was not taking any medications known to cause thrombocytopenia and infection was excluded. A diagnosis of ITP was made by exclusion and the patient was started on eltrombopag $50 \mathrm{mg}$ daily. One week later, her platelets increased to 100 and then to 279 in 2 weeks period. So eltrombopag was decreased to $25 \mathrm{mg}$ daily and platelet count was checked after 1 week of dose reduction and found to be 182. In late 2015, the patient decided to get pregnant and she decided to stop eltrombopag after she knew that its effect on pregnancy was not well recognized. In 2019, she presented with platelet count 21 and she was asymptomatic. Again, full workup was done including complete blood count and inflammatory markers, which was normal. Medication-induced thrombocytopenia was excluded and peripheral smear showed reduced platelet number. Eltrombopag $25 \mathrm{mg}$ daily was started. After 1 week, platelet count increased to 116 and 1 week later to 244 . After 2 weeks of initiation of eltrombopag, the dose was reduced to $25 \mathrm{mg}$ every other day and she maintain platelet count of 168 after 1 week. The patient was followed over the next few months and her platelet count is shown in Fig. 1. 
Ahmed/Yassin: Eltrombopag in Chronic ITP Post Sleeve Gastrectomy

\section{Discussion/Conclusion}

The two medications commonly used as second line for ITP are rituximab and eltrombopag. Rituximab is a chimeric mouse/human monoclonal antibody that binds specifically to the transmembrane antigen CD20 [6, 7]. Eltrombopag, an oral thrombopoietin receptor agonist, is approved in the Middle East, Turkey, Asia, and Australia for the treatment of thrombocytopenia in adults with chronic immune thrombocytopenia (cITP) with insufficient response to prior therapy, such as corticosteroids and immunoglobulins. To date, real-world data on eltrombopag use in patients with cITP have not been collected in a systematic manner in most countries outside the US and Europe. Eltrombopag is absorbed with a peak concentration occurring 2-6 $\mathrm{h}$ after oral administration. Administration of eltrombopag concomitantly with antacids, dairy products, mineral supplements, or other products containing polyvalent cations significantly reduces eltrombopag exposure. The absolute oral bioavailability of eltrombopag after administration to humans has not been established. Based on urinary excretion and metabolites eliminated in feces, the oral absorption of drug-related material following administration of a single 75-mg eltrombopag solution dose was estimated to be at least $52 \%$ [8]. Eltrombopag is primarily metabolized through cleavage, oxidation, and conjugation with glucuronic acid, glutathione, or cysteine [7]. Absorbed eltrombopag is extensively metabolized. The predominant route of eltrombopag excretion is via feces (59\%) with 31\% of the dose found in the urine as metabolites. Unchanged parent compound (eltrombopag olamine) is not detected in urine. Unchanged eltrombopag olamine excreted in feces accounts for approximately $20 \%$ of the dose. The plasma elimination half-life of eltrombopag is approximately 21-32 $\mathrm{h}$ [7].

For eltrombopag dose adjustment, the lowest dose should be used to achieve and maintain platelet count $\geq 50,000 / \mathrm{mm}^{3}$ as needed to reduce the risk of bleeding. It is to be discontinued if platelet count does not respond to a level that avoids clinically important bleeding after 4 weeks at the maximum of $75 \mathrm{mg} /$ day [8].

Initial dose is $50 \mathrm{mg}$ once daily ( $25 \mathrm{mg}$ once daily for patients of Asian ethnicity, e.g., Chinese, Japanese, Korean, Taiwanese); the dose should be titrated based on platelet response. Maximum dose is $75 \mathrm{mg} /$ day [8].

\section{Dosage Adjustment Based on Platelet Response}

Platelet count $<50,000 / \mathrm{mm}^{3}$ ( $\geq 2$ weeks after treatment initiation or a dose increase): increase daily dose by $25 \mathrm{mg}$ (if taking $12.5 \mathrm{mg}$ once daily, increase dose to $25 \mathrm{mg}$ once daily prior to increasing the dose amount by $25 \mathrm{mg}$ daily); maximum: $75 \mathrm{mg} /$ day [8].

Platelet count $\geq 200,000 / \mathrm{mm}^{3}$ and $\leq 400,000 / \mathrm{mm}^{3}$ (at any time): reduce daily dose by 25 $\mathrm{mg}$ (if taking $25 \mathrm{mg}$ once daily, decrease dose to $12.5 \mathrm{mg}$ once daily); reassess in 2 weeks [8].

Platelet count $>400,000 / \mathrm{mm}^{3}$ : withhold dose; assess platelet count twice weekly; when platelet count $<150,000 / \mathrm{mm}^{3}$, resume with the daily dose reduced by $25 \mathrm{mg}$ (if taking $25 \mathrm{mg}$ once daily, resume with $12.5 \mathrm{mg}$ once daily) [8].

Platelet count $>400,000 / \mathrm{mm}^{3}$ after 2 weeks at the lowest dose: discontinue treatment [8].

So, in our case the patient started initially on $25 \mathrm{mg}$ daily. Then in 2 weeks duration, her platelets increased to 244 , then the dose was reduced to $12.5 \mathrm{mg}$ daily and she maintained platelets, although she had sleeve gastrectomy, which will reduce availability of the drug.

\section{Conclusion}

It seems that from our case, eltrombopag is not affected by sleeve gastrectomy as well as the role of ethnicity in response to thrombopoietin agonist, which should be taken into consideration; however, further studies are needed to confirm this finding. 


\section{Acknowledgement}

Authors acknowledge internal medicine residency program for scientific support and Qatar national library for financial support.

\section{Statement of Ethics}

Case was approved by the HMC medical research center and the subject has given her written informed consent to publish her case.

\section{Disclosure Statement}

Both authors declare no conflict of interest.

\section{Funding Sources}

Qatar national library.

\section{Author Contributions}

Both authors contributed equally.

\section{References}

1 Gonzalez-Porras JR, Escalante F, Pardal E, Sierra M, Garcia-Frade LJ, Redondo S, et al. Safety and efficacy of splenectomy in over 65-yrs-old patients with immune thrombocytopenia. Eur J Haematol. 2013 Sep; 91(3):236-41.

2 Cines DB, Blanchette VS. Immune thrombocytopenic purpura. N Engl J Med. 2002;346(13):995-1008.

3 Rodeghiero F, Stasi R, Gernsheimer T, et al. Standardization of terminology, definitions and outcome criteria in immune thrombocytopenic purpura of adults and children: report from an international working group. Blood. 2009;113(11):2386-2393.

4 Cuker A. Transitioning patients with immune thrombocytopenia to second-line therapy: Challenges and best practices. Am J Hematol. 2018;93(6):816-23.

5 Eltrombopag (Promacta®), OncoLink [Internet]. [cited 2020 Mar 3]. Available from: https://www.oncolink. org/cancer-treatment/oncolink-rx/eltrombopag-promacta-r

6 Rituxan P. Product Monograph [Internet]. 2000 [cited 2020 Mar 8]. Available from: www.rochecanada.com

7 Yassin M, El-Ayyoubi H, Muhammed H, Kamzoul R, Al-Badri M, Al-Sabbah A. Efficacy and safety of six doses of rituximab $\left(375 \mathrm{mg} / \mathrm{m}^{2}\right)$ in treatment of chronic refractory immune thrombocytopenia an experience from Qatar. Haematologica. 2011;96:648-9.

8 Eltrombopag: Drug information - UpToDate [Internet]. [cited 2020 May 27]. Available from: https://www. uptodate.com/contents/eltrombopag-drug-information?search=eltrombopag\&source=panel_search_ result\&selectedTitle=1 16\&usage_type=panel\&kp_tab=drug_general\&display_rank=1. 